\title{
THE SPREAD OF EVIDENCE-POOR MEDICINE VIA FLAWED SOCIAL-NETWORK ANALYSIS
}

\author{
RUSSELL LYONS
}

Dedicated to the memory of David A. Freedman

\begin{abstract}
The chronic widespread misuse of statistics is usually inadvertent, not intentional. We find cautionary examples in a series of recent papers by Christakis and Fowler that advance statistical arguments for the transmission via social networks of various personal characteristics, including obesity, smoking cessation, happiness, and loneliness. Those papers also assert that such influence extends to three degrees of separation in social networks. We shall show that these conclusions do not follow from Christakis and Fowler's statistical analyses. In fact, their studies even provide some evidence against the existence of such transmission. The errors that we expose arose, in part, because the assumptions behind the statistical procedures used were insufficiently examined, not only by the authors, but also by the reviewers. Our examples are instructive because the practitioners are highly reputed, their results have received enormous popular attention, and the journals that published their studies are among the most respected in the world. An educational bonus emerges from the difficulty we report in getting our critique published. We discuss the relevance of this episode to understanding statistical literacy and the role of scientific review, as well as to reforming statistics education.
\end{abstract}

\section{INTRODUCTION}

For at least 130 years, it has been common knowledge that statistics are widely abused. Less well known among the public is that professional publications even in top medical journals routinely, though unwittingly, misuse statistics. The corollary that top journals do not serve as rigorous judges of quality, due to lack of statistical competence, is not often discussed.

We illustrate the latter two themes in this paper by presenting some cautionary examples of somewhat sophisticated recent statistical analyses that were flawed by insufficient attention to assumptions and misinterpretation of results. Novel techniques were used to analyze social networks. The results of these analyses were published in the most respected medical journals and have become rather famous, even outside academia. However, both elementary statistical errors and more advanced errors undermine these analyses to such an extent that little can be deduced from the original studies - except that we need to improve our statistics education. Despite medicine's recent emphasis on improving the nature of their evidence, the medical field still has a long road ahead.

We hope that our analysis will be useful to educators, to practitioners, and to all who have an interest in the quality of scientific research that relies on statistics. With such audiences in mind, we have endeavored to explain our analysis as carefully as possible, while minimizing mathematical derivations.

The statistics in question come from a series of recent papers $[1,2,3,4]$ by Christakis and Fowler (C\&F), who analyzed network data coming from the Framingham Heart Study. This long-running observational study collects not only physical health information, but also other personal characteristics, including elements of the social network of participants. C\&F analyzed new data via new statistical techniques, leading to two major inferences:

(1) There is a process of infection or contagion within this social network that transmits various personal characteristics, including obesity, smoking cessation, happiness, and loneliness.

Date: 4 May 2011.

Department of Mathematics, 831 E. 3rd St., Indiana University, Bloomington, IN 47405-7106, USA. rdlyons@indiana.edu, http://mypage.iu.edu/ ${ }^{\sim}$ rdlyons. 
(2) Such transmission occurs up to three steps in the network, providing evidence of a universal " "three degrees of influence' rule of social network contagion" [4].

C\&F's studies have received considerable acclaim in the popular press and in society at large. For example, their study on obesity was reported on the front page of The New York Times, above the fold, and was at some time e-mailed from the website more than any other article but one that day. Both authors were named one of the "Top 100 Global Thinkers" in 2010 by Foreign Policy magazine. Rudolph Leibel, a member of the Institute of Medicine of the National Academy of Sciences, said of C\&F's paper [1] on obesity that "It is an extraordinarily subtle and sophisticated way of getting a handle on aspects of the environment that are not normally considered" [5]. Daniel Kahneman, a Nobel-prize winner, said of C\&F's paper [3] on happiness that "It's extremely important and interesting work" [6]. Considerable professional success has attended their work, with large grants coming their way; the largest to date is for $\$ 11,000,000$ from the National Institute of Aging. Their conclusions have also been disseminated via a popular book [7], which has been translated into twenty languages.

Despite such accolades, we shall establish that both of their major claims are unfounded. That is, while the world may indeed work as C\&F say, their studies do not provide evidence to support such claims. Moreover, parts of their studies even suggest that their claims of transmission are untrue.

In the remainder of this introduction, we present a summary of their evidence and a summary of our arguments against it. Later sections provide details.

All of C\&F's papers in this series use similar methods, so for brevity, we refer only to their obesity study. The Framingham Heart Study has about 12,000 participants, who are examined every few years. About 5,000 of the participants are in the "Offspring Cohort". It is those in the Offspring Cohort whose obesity is analyzed in relation to the obesity of all 12,000 participants.

C\&F start by finding statistical associations between the obesity of friends in the Framingham network: To oversimplify, a person's friends are more likely to be obese if the person himself is obese. The associations that $\mathrm{C} \& \mathrm{~F}$ analyze are calculated from statistical models whose parameters are estimated by using the observational data. This source of C\&F's associations is crucial to their argument and decisive for our critique.

C\&F argue that these associations are not mere associations, but measure causal effects. The two primary reasons the associations might not be causal are homophily (or selection), which is the fact that people tend to associate with others like themselves, and a shared environment (also called "confounding" or "contextual influences" by other researchers). C\&F call the causal effects induction (also called "influence" or "endogenous social effect" by others) that they liken to a transmission process. C\&F deduce induction indirectly by ruling out the possibilities of homophily and shared environment; they provide speculation, but not evidence, for how such induction might work.

For concreteness in our explanations, suppose that Frank is a study participant in the Offspring Cohort. C\&F use a logistic regression to model the probability of Frank's obesity at a given exam. The important variables used in the model are Frank's obesity status at the previous exam and the obesity status-both now and at the previous exam - of those (such as, say, Linda) to whom Frank is connected in his social network.

C\&F argue against the homophily explanation because their logistic regression model included a term for Linda's obesity status at the previous exam. Since the model produces the associations to be studied, they are supposed to be net of any effects of homophily.

C\&F argue against the shared environment explanation as follows. Consider two friends, Frank and Linda. C\&F have "directional" information on friendships: Each participant was asked to name one close friend. Suppose Frank named Linda as his "closest" friend, but not vice versa. C\&F find that if Linda becomes obese, then Frank's chance of becoming obese himself increases by $57 \%$ relative to what it would be if Linda did not become obese. On the other hand, suppose that Linda named Frank as her closest friend, yet Frank did not name Linda as his closest friend. In this case, if Linda becomes obese, then Frank has only a $13 \%$ increased chance of becoming obese. Since $57 \%$ is far different from $13 \%$, C\&F contend that this asymmetry rules out a shared environment between Frank and Linda as a cause of their associated obesity. C\&F conclude that 
having accounted for or ruled out the other possible explanations for the observed associations in obesity, it must be induction that produces these associations.

In order to establish their three-degrees-of-influence rule, $\mathrm{C} \& \mathrm{~F}$ compare the network data they have to random networks, where they change who is obese, while maintaining the existing social ties. By comparing statistical associations in the actual network to those in the random networks, they find that obesity is significantly associated out to three degrees and not further.

While the influence of friends' obesity on others depends on social distance in this way, according to C\&F, it does not depend on geographic distance, even when the friends involved rarely see each other. C\&F also say that obesity spreads to a friend of a friend (or even to a friend of a friend of a friend) without the intermediate friend(s) becoming obese [8].

However, the arguments given to substantiate C\&F's claims are not sound, primarily because of two kinds of errors:

(1) C\&F use statistical models that contradict their data, as well as their conclusions.

(2) Even if one accepts C\&F's statistical models and tests, C\&F interpret the results incorrectly.

As we have noted, the increases in obesity risk reported above do not arise from calculations based directly on the data. Rather, they arise indirectly from the data: They result from statistical models that were fitted to the observational data. By the nature of a statistical model, the numbers above, $57 \%$ and $13 \%$, come with uncertainties. C\&F say that these numbers are statistically distinguishable. However, when we look more closely (in critique (1) of Section 2), we shall see that they are in fact not distinguishable - due to the large uncertainties inherent in them. We shall also demonstrate (in critique (2) of Section 2) that their addition of a lagged obesity term in their models does not properly control for homophily; rather than subtract the effect of homophily, if anything, it amplifies it. Moreover, a closer examination of the idea of directional associations will show (in critique (3) of Section 2) that the proposed differences are actually consistent with all three types of explanation: homophily, shared environment, and induction. In sum, C\&F have not shown that they can distinguish among the three possible explanations.

We shall examine the first category of error in Section 4; as it is the most technical aspect of our analysis, we reserve it for last. C\&F's statistical models will turn out to have serious problems due precisely to the network effects C\&F hope to analyze. For example, the asymmetry discussed above, produced by their model and intended to rule out a shared-environment explanation, turns out to be mathematically inconsistent with their model. How can the model produce a result that is inconsistent with itself? It is because C\&F's method of estimation of their model is inapplicable to their model. All these problems cast doubt on C\&F's reported numbers. Moreover, as noted above, C\&F provide other evidence that associations persist in the face of geographic separation; this suggests that homophily is, in fact, playing the major role. In our view, the most important task of $\mathrm{C} \& \mathrm{~F}$ is to show that homophily does not explain their associations. For a simple example showing how homophily relates to shared changes in health, suppose that Sally gets cancer. Then her friends are more likely to have gotten cancer than those who are not Sally's friends. Why? Because Sally is likely old and so are her friends. (Of course, in this example, one can control for age. The difficulty in general is to control properly for all confounding factors, including the unknown ones.)

It is true that the three-degree rule exists in the network data that C\&F use. However, this is partly due to the nature of their data, which is sparse. For example, in many cases, friends of friends will be friends, but this is not recorded in their data. The network assembled from this data, therefore, is likely to mislead.

Following our critique of C\&F's work, we consider the implications for quality control at top journals in Section 6. We also describe briefly the difficulties we had in getting our critique published and the attitudes we encountered from top journals towards critiques. In our last section, we place this episode in a general context of a misplaced faith in statistical models, illustrated with quotes from distinguished critics. We urge that statistics education place much more emphasis on critical thinking. 


\section{Directionality}

We begin with a critique of C\&F's argument against the shared environment explanation. Their argument is based on perceived directional differences in friendships. To understand the issues, we must review a key trait of their studies.

As we have said, certain participants, the Offspring Cohort, are chosen to be the focus of analysis; they are called "focal participants" (abbreviated FP) in [4], and are called "egos" in the other studies. The participants to whom they are linked by a tie of friendship, family, workplace, or neighborhood are called "linked participants" (abbreviated LP) in [4], and called "alters" in the other studies. Some LPs are also FPs. Thus, FP is an absolute term, while LP is relative to the FP. As we said above, each participant was asked to list one close friend. (Some people listed more than one, despite the instructions.) The friendship data in the Framingham Heart Study consists of the record of those answers. This leads to the key property that friendship ties are directional, from FP to LP or from LP to FP. In case each names the other, then the tie goes both ways. Some ties are between two LPs, neither of whom is an FP, so those ties are not included in most of C\&F's analyses. Furthermore, only ties to people who also were in the Framingham Heart Study were included in C\&F's analyses. In the case of friends, for example, those included amounted generally to less than $1 / 4$ of all named friends: see [2, supplement, Table S2].

Here is how $\mathrm{C} \& \mathrm{~F}$ explain the directional differences in [1]:

If an ego stated that an alter was his or her friend, the ego's chances of becoming obese appeared to increase by $57 \%$ (95\% confidence interval [CI], 6 to 123) if the alter became obese. However, the type of friendship appeared to be important. Between mutual friends, the ego's risk of obesity increased by $171 \%$ (95\% CI, 59 to 326) if an alter became obese. In contrast, there was no statistically meaningful relationship when the friendship was perceived by the alter but not the ego $(\mathrm{P}=0.70)$. Thus, influence in friendship ties appeared to be directional.

$\cdots$

the findings regarding the directional nature of the effects of friendships are especially important with regard to the interpersonal induction of obesity because they suggest that friends do not simultaneously become obese as a result of contemporaneous exposures to unobserved factors. If the friends did become obese at the same time, any such factors should have an equally strong influence regardless of the directionality of friendship. This observation also points to the specifically social nature of these associations, since the asymmetry in the process may arise from the fact that the person who identifies another person as a friend esteems the other person.

In order to discuss this argument, it will be useful to abbreviate a friendship tie as $\mathrm{FP} \rightarrow \mathrm{LP}$ when the FP named the LP but the LP did not name the FP; LP $\rightarrow \mathrm{FP}$ when the LP named the FP but the FP did not name the LP; and $\mathrm{FP} \leftrightarrow \mathrm{LP}$ when the naming was mutual. Thus, C\&F are saying that causality is the best explanation for the differences among $171 \%$ for $\mathrm{FP} \leftrightarrow \mathrm{LP}, 57 \%$ for $\mathrm{FP} \rightarrow \mathrm{LP}$, and $13 \%$ for $\mathrm{LP} \rightarrow \mathrm{FP}$.

We claim that C\&F's argument from directional differences has the following three problems, ${ }^{1}$ which we discuss in turn:

(1) The differences are not statistically significant.

(2) C\&F's argument that the differences are net of homophily is incorrect.

(3) The differences are consistent with all three possible explanations.

(1) The first problem is that the differences are not statistically significant. Let us consider carefully their reasoning: $\mathrm{C} \& \mathrm{~F}$ estimate an $\mathrm{FP} \rightarrow \mathrm{LP}$ increased obesity risk of $57 \%$ and an $\mathrm{LP} \rightarrow \mathrm{FP}$ increased obesity risk of $13 \%$. However, they accept that their estimates are not precise. They feel $95 \%$ confident that the former lies in the interval from $6 \%$ to $123 \%$, while the latter, being statistically insignificant, might well be $0 \%$. Since $0 \%$ does not lie in the interval $[6 \%, 123 \%]$, they infer that the two risks are different. But this reasoning exemplifies a statistical error that is common in many studies and that occurs throughout C\&F's (Section B of the Appendix). The error is to mistake a number for 0 when one has learned only that the available evidence is too imprecise to distinguish the number from 0 . In the present case, the estimate $13 \%$

\footnotetext{
${ }^{1}$ Versions of these three problems were mentioned briefly in the editorial [9]. The latter two were also discussed in the letter [10]. A theoretical discussion related to the second point, whether it is even possible to control for homophily without making assumptions, is given by [11].
} 
for the $\mathrm{LP} \rightarrow \mathrm{FP}$ risk has a CI that seems to be $[-28 \%, 68 \%]$. C\&F take the "true value" to be $0 \%$, but there is no reason to take the "true value" to be $0 \%$. Their estimate is $13 \%$ and $13 \%$ itself falls in the CI for the $\mathrm{FP} \rightarrow \mathrm{LP}$ risk. To compound the error, $57 \%$ also falls in the CI for the $\mathrm{LP} \rightarrow \mathrm{FP}$ risk. This means that $\mathrm{C} \& \mathrm{~F}$ 's numbers do not distinguish the associations in the two directions. The observed differences could be due to chance, according to C\&F's technique.

This same error regarding statistical significance for directional estimates occurs in each of C\&F's papers; it is summarized in Figure 1. (See Table 1 of Appendix A for the numerical estimates and intervals.)

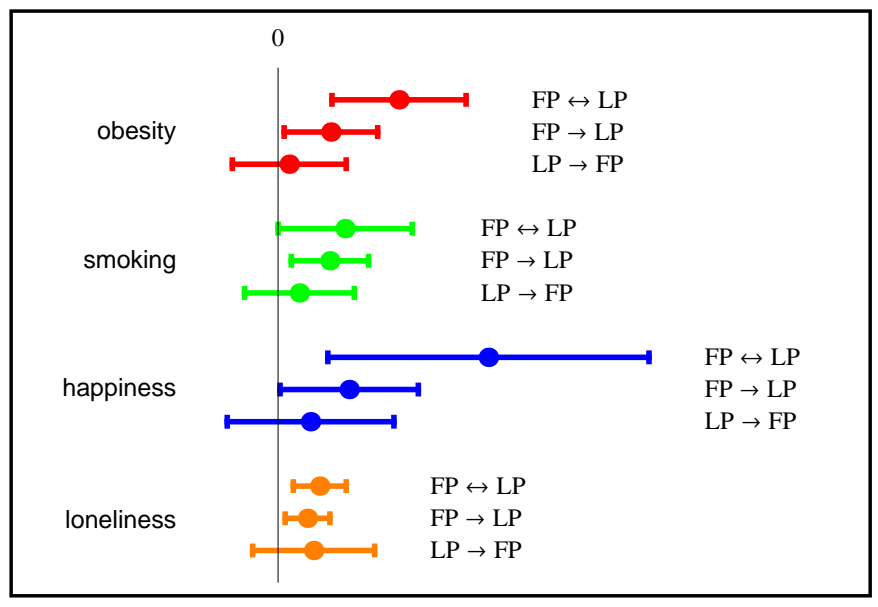

Figure 1. Coefficient estimates and $2 \mathrm{SE}(95 \%)$ confidence intervals for directional effects. For each study, the order from top to bottom is (1) mutual friendship, (2) FP named LP, then (3) LP named FP. The CIs overlap so much that one cannot infer that the differences are statistically significant. Sources: [1, suppl. p. 3]; [2, suppl. p. 18]; [3, suppl. p. 9]; [4, pp. 983-984].

A technical note: $\mathrm{C} \& \mathrm{~F}$ are comparing coefficients from different models. Therefore, they cannot estimate the difference between these coefficients. They would be able to make an inference on the difference of two coefficients if they had a valid model that contained both coefficients. We don't know such a model and, for the general reasons discussed in Section 7, we are skeptical that one exists. Putting such skepticism aside, if we wished to construct such a model, we would need access to the data. The social network data for the Framingham Heart Study was assembled by C\&F from hand-written data, but C\&F have not made this available to others. This also prevents the most basic type of replication [12] and can keep errors hidden [13]. In any case, given what C\&F have, they do not have reason to infer that these differences are statistically significant.

(2) Suppose we ignore this inferential difficulty and allow C\&F their directional differences; after all, there is indeed a clear pattern in the estimates. According to C\&F, these differences rule out confounding. What about homophily? C\&F counter this explanation as follows. The numbers above (such as 57\% and 13\%) arise from logistic regression models. C\&F [1] say, "Our models account for homophily by including a time-lagged measurement of the alter's obesity." That is, in the equations predicting the FP's current obesity, there is a variable that indicates whether the LPs were obese in the previous exam. Therefore, the risks that C\&F analyze are supposed to be net of whatever effects may be due to homophily. C\&F do not give a separate argument against homophily. Their reasoning hinges, then, on whether the lagged term properly controls for homophily. Let us look.

Their model has two related terms, one for the LP's current obesity and the other for the LP's lagged obesity, i.e., the LP's obesity status at the previous exam. The current obesity is used to measure "effect" on the FP's obesity, while the lagged obesity is used to "control" for homophily. One might argue that the reverse (if either) should be used, as causal effects require a time difference. However, either choice leads to disquiet when we use the estimates $\mathrm{C} \& \mathrm{~F}$ give for the two corresponding coefficients, as these two coefficients 
sum to approximately 0 [1, suppl., Tables S1, S2, S3]. In particular, they have opposite signs. Thus, if the lagged obesity were used for "effect", we would conclude that the social network inhibits the spread of obesity, while if the lagged obesity were used for "control", as C\&F do, then we would be left with the puzzle that homophily affects the FP and the LP in opposite ways. Should we find such opposite effects too unsettling, then to the extent that the lagged term relates to homophily, we would conclude that rather than taking away the effect of homophily, the term has amplified its effect.

We remark that Cohen-Cole and Fletcher [14, 15] also felt that C\&F had not controlled properly for homophily. They attempted to show the unreliability of C\&F's work by deducing implausible conclusions from similar modeling techniques and by showing how the conclusions change with different controls. C\&F [16, full version at the authors' websites] responded by noting that their critics found only statistically insignificant results and by a simulation. Another difficulty in C\&F's work was pointed out by [17]: When friendships change in ways related to homophily, then estimates of the effects of variables other than homophily can be biased.

(3) The third problem is that directional differences are actually consistent with all three considered explanations, i.e., induction, homophily, and environment. Consider the three types of ties, FP $\leftrightarrow \mathrm{LP}, \mathrm{FP} \rightarrow \mathrm{LP}$, and $\mathrm{LP} \rightarrow \mathrm{FP}$, and, for each type, the possible correlations of the LP's obesity with the obesity of the FP. As shown in Figure 1, C\&F find that the strength of these three correlations are different and in order of most to least. They say that this eliminates the possibility that these correlations are due to a shared environment. We say that one expects this same ordering of strength of correlation whether the correlations are due to induction, homophily, or even shared environment. Furthermore, this is true regardless of whether one finds these correlations due to modeling or other reasons: this is a general phenomenon arising from the choice of whom to correlate with the FP. C\&F have argued the case for induction (who "esteems" whom); we explain why the same holds for homophily and shared environment.

Consider the following hypothetical situation. Imagine that each individual names as a friend the other person whose characteristics (covariates) are objectively closest to his own. See Figure 2 for a representation, to be explained further below. If we are thinking about homophily, then this naming process represents people selecting each other based on similar characteristics. If we are thinking about shared environment, on the other hand, then this process represents people making friends with their closest neighbors.

One of the individuals is Frank, an FP, who names Linda, an LP, as his friend. How correlated is Frank's obesity with Linda's as a function of their type of friendship? By definition, Linda is closer to Frank than anyone else, including those who named Frank as their closest friend. If distance represents degree of homophily, then this means Linda is more like Frank than anyone else, while if distance arises from location, then Linda shares more of Frank's environment than anyone else. In each of these two cases, Linda is more correlated with Frank than are those others who name Frank (the LP $\rightarrow$ FP ties). Finally, if it happens that Linda also named Frank reciprocally (so their tie is of FP $\leftrightarrow$ LP type), then this pair of individuals is especially close to each other and thus Linda is even more correlated with Frank. Thus, we see in this hypothetical situation precisely the kind of directional differences C\&F find: FP $\leftrightarrow$ LP ties have the strongest associations, followed by $\mathrm{FP} \rightarrow \mathrm{LP}$ and then $\mathrm{LP} \rightarrow \mathrm{FP}$. In sum, the directional differences C\&F found are just what one would expect to see for all three types of explanations; the differences do not distinguish among the explanations.

We now discuss Figure 2 in order to elucidate why mutual friends are especially close to each other. Consider the following chance model for the above hypothetical situation. Let $B$ be a ball in a highdimensional space. The location of an individual in $B$ represents various of his covariates. Imagine that individuals are independently uniformly distributed in $B$. Now each person names one other as a friend, namely, that person who is closest to him. Figure 2 shows this in two dimensions. One can easily prove mathematically that the distance between mutual friends is stochastically smaller than the distance between non-mutual friends. (This means that for every number $d$, the probability that the distance between mutual friends is less than $d$ is at least the probability that the distance between non-mutual friends is less than $d$.) Thus, mutual friends are generally closer to each other than are non-mutual friends, as is apparent visually in the figure. 


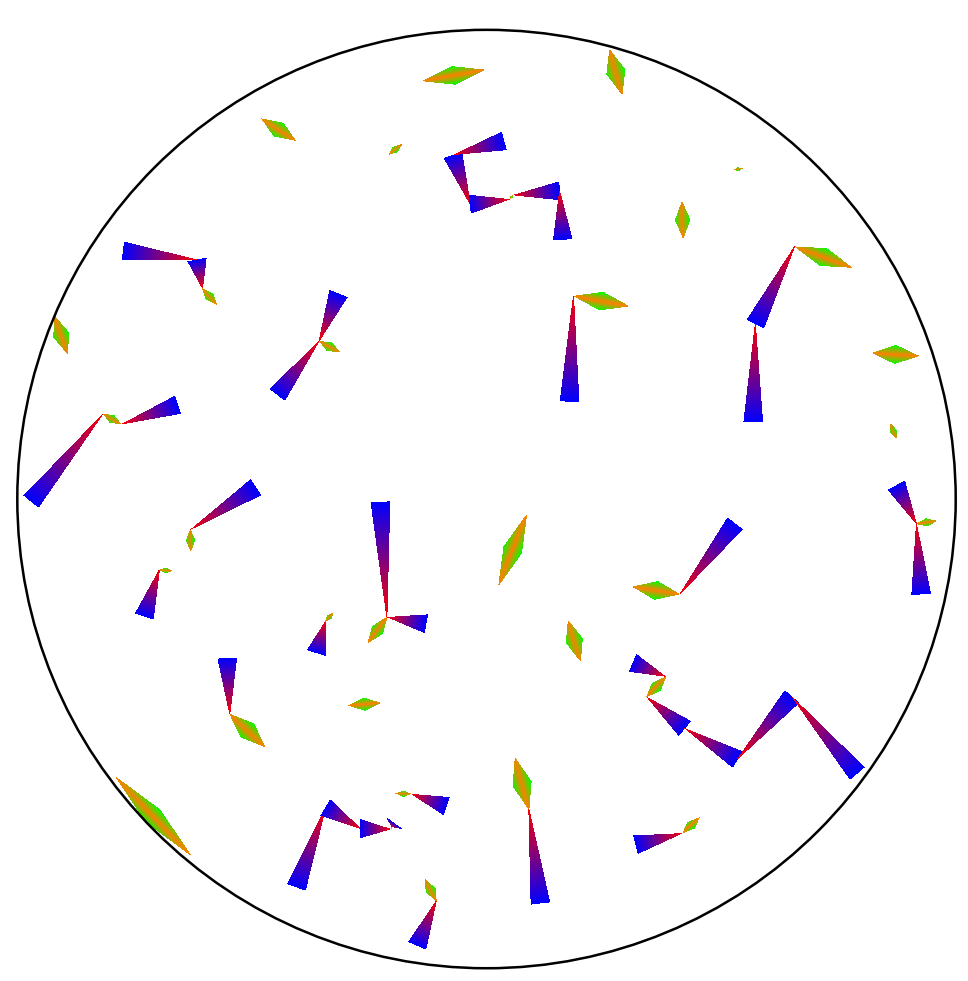

directional tie:

mutual tie:

Figure 2. 100 random locations in a disc, each pointing to its nearest neighbor. Locations that point to each other are usually especially close to each other.

\section{RANDOM NETWORKS}

We now consider the methods and meanings of C\&F's statistical calculations. They use two methods across their papers: one consists of varying values in the given network, while the second consists in making regressions. The first method leads to C\&F's three-degrees-of-influence rule, while the second method leads to the estimates and CIs discussed in the preceding section. Although it would be logical to discuss the regressions now, they are much more technical, so we defer that discussion until after we discuss the random networks in this section.

In [1], the authors preserve the network, but randomly redistribute the incidence of obesity (preserving the same number of obese individuals). By comparing the actual network to the randomly generated networks, $\mathrm{C} \& \mathrm{~F}$ demonstrate that statistical associations of obesity between pairs of people extend to three degrees of separation in the observed network. The same result holds for smoking cessation, happiness, and loneliness. This is a reasonable method to summarize the structure that exists in the observed network as it relates to the characteristic of interest.

However, as we noted already, the data used by $\mathrm{C} \& \mathrm{~F}$ is incomplete and thus the network treats some people as not friends when in reality they are friends. Only $45 \%$ of the $5124 \mathrm{FPs}$ named a friend in the Study. There were 3604 unique observed friendships in total $[1,3]$, but not all were among those named at any one time. This means that the average current number of friends reported was about 0.7 per FP. Thus, the network data concerning friends, in particular, is quite thin. Therefore, the three-degree pattern, while present in the network assembled from the data used, has not been demonstrated for the real world.

This random-network analysis is unrelated to the cause of the statistical associations; C\&F turn to regression models to argue their causal conclusions. But since $\mathrm{C} \& \mathrm{~F}$ find in $[1,2]$ that the associations in their networks are essentially unrelated to geographic distance, they have in fact given evidence that the 
associations of obesity and smoking are due to homophily, more than to a shared environment, and unlikely due to induction.

A further problem is that the language $\mathrm{C} \& \mathrm{~F}$ use blurs the distinction between the model and reality. For example, [1] reports that "the risk of obesity among alters who were connected to an obese ego (at one degree of separation) was about $45 \%$ higher in the observed network than in a random network." ${ }^{2}$ In [4], C\&F write that "a person's loneliness depends not just on his friend's loneliness but also extends to his friend's friend and his friend's friend's friend. The full network shows that participants are $52 \%$ (95\% CI $=40 \%$ to $65 \%$ ) more likely to be lonely if a person to whom they are directly connected (at one degree of separation) is lonely." This sounds very much like predicting what would happen in the real world if a friend became lonely, which, after all, is a main goal of the paper. Indeed, C\&F frame this particular figure in terms of "the 'three degrees of influence' rule of social network contagion that has been exhibited for obesity, smoking, and happiness" [4]. Further reinforcing the idea that C\&F are making a prediction, the CI appears to quantify the uncertainty in the prediction. However, this $52 \%$ is not a risk of friendship, nor is it a prediction about the real world, nor did it involve comparisons across time. It is merely a numerical comparison of the observed network to a certain random network. Likewise, the CI is not actually a confidence interval: Confidence intervals aim to contain the true unknown parameter and are obtained by random sampling from the true population, whereas here, we know the real network and randomly sample the imaginary network, which, by design, is not realistic. This blurring of model and reality can mislead readers. Accordingly, the editorial [18] commented on [3] that "the size of the influence of distant friends (friends of friends' friends; $5.6 \%$ ) seems overly large when the influence of a happy friend is only 14\%." The figures quoted here by [18] come directly from C\&F's random networks and so do not represent influences.

\section{Modeling}

The bulk of the numbers produced by $\mathrm{C} \& \mathrm{~F}$ arise from an abundance of logistic or linear regression models, intended to describe and explain the observed associations. $\mathrm{C} \& \mathrm{~F}$ have not done an experiment, nor run a so-called natural experiment, and they do not have enough data for multi-dimensional cross-tabulation; this is why they turn to statistical models [19].

Use of a statistical model requires $\mathrm{C} \& \mathrm{~F}$ to make assumptions about what the data would look like if either they had an experiment or they had much more data. If these assumptions are wrong, then C\&F's conclusions may be invalid or misleading. C\&F pay very little attention to their assumptions, but they are crucial for the validity of their methods. We shall examine only a few of those assumptions here. Notably, we shall find that their regression models contradict their data and their conclusions about directional effects.

In order to see clearly what $\mathrm{C} \& \mathrm{~F}$ assume, it is important to describe precisely their models. The first time that C\&F stated their models was in [20], where they were invited to discuss the statistical foundations for their work. $\mathrm{C} \& \mathrm{~F}$ aim to reveal causation by technical means (they do not claim to have observed an induction mechanism), and only a technical examination can reveal fully the flaws.

Let $Y_{i, t}$ be the indicator that individual $i$ is obese at time $t$. (An indicator is 1 if the event is true, and 0 otherwise.) These times can be any integer from 1 to 7 ; they correspond to exam periods (called "waves"), which occurred every few years. Let $W_{n}(t)$ be the indicator that $t=n$; let $A_{i, t}$ be the age of $i$ in years at time $t$; let $F_{i}$ be the indicator that $i$ is female; and let $E_{i, t}$ be the number of years of education of $i$ at time $t$. Abbreviate by $C_{i, t}$ the collection of indicator variables $Y_{j, s}$ for all pairs $(j, s) \neq(i, t)$. Different models arise by considering various sets $T_{t}$ of ties at times $t$, as well as by changing the covariates listed above. For given sets $T_{2}, T_{3}, T_{4}, T_{5}, T_{6}$, and $T_{7}\left(T_{t}\right.$ might equal, say, all mutual ties among FPs that existed at both times $t$ and $t-1), \mathrm{C} \& \mathrm{~F}$ posit a system of simultaneous equations for the joint distribution of $Y_{i, t}$ : there are

\footnotetext{
${ }^{2}$ The caption of their Fig. 3 differs from the text as to what they measured. The caption interchanges "alter" and "ego" and reports this as "Relative Increase in Probability of Obesity in an Ego if Alter becomes Obese". The same conflict of description occurs with Fig. 2 in [2].
} 
some numbers $\alpha, \beta_{k}, \gamma_{n}, \delta_{k}$ such that for all $2 \leq t \leq 7$ and all $(i, j) \in T_{t}$, we have ${ }^{3}$

$$
\log \frac{\mathbf{P}\left[Y_{i, t}=1 \mid C_{i, t}\right]}{\mathbf{P}\left[Y_{i, t}=0 \mid C_{i, t}\right]}=\alpha+\left(\beta_{1} Y_{j, t}+\beta_{2} Y_{j, t-1}\right)+\beta_{3} Y_{i, t-1}+\sum_{n=3}^{7} \gamma_{n} W_{n}(t)+\delta_{1} A_{i, t}+\delta_{2} F_{i}+\delta_{3} E_{i, t}
$$

The two terms in parentheses on the right-hand side are the key terms. C\&F's main interest is in estimating $\beta_{1}$, which they call the "effect" of the LP's current obesity on the FP's current obesity. The summand $\beta_{2} Y_{j, t-1}$ is supposed to control for homophily. Change in obesity is represented by having the FP's obesity status at the previous exam, $Y_{i, t-1}$, on the right-hand side of the equation. The rest of the covariates on the right-hand side are supposed to control for time and personal characteristics.

For example, for mutual friends, $\mathrm{C} \& \mathrm{~F}$ estimate $\beta_{1}=1.19$ with an SE of 0.33, which they translate to an increased risk of $171 \%$ with a $95 \%$ CI of $[59 \%, 326 \%]$.

A fundamental problem is that there are too many equations: one for each tie. That's usually more than the number of $Y_{i, t}$. If $i$ names more than one $j$ at time $t$, then $\left(\beta_{1} Y_{j, t}+\beta_{2} Y_{j, t-1}\right)$ must be the same for all such $j$ because that's the only thing that changes in the equation when $j$ changes. Thus, the model contradicts the data - unless $\beta_{1}=\beta_{2}=0$, in which case no individual affects any other at any time.

In fact, it turns out that $\beta_{1}=0$ regardless of the data. ${ }^{4}$ That is, C\&F's model contradicts their conclusions concerning directionality. To see this, let the set $T_{t}$ of ties consist of FP $\rightarrow$ LP ties (as C\&F do for estimating the "risk" of $\mathrm{FP} \rightarrow \mathrm{LP}$ ties). Suppose $(i, k),(k, m) \in T_{t}$, where $m \neq i$. Let $D_{i, k, t}$ be the collection of indicator variables $Y_{j, s}$ for all pairs $(j, s) \neq(i, t),(k, t)$. We may calculate $\log \left(\mathbf{P}\left[Y_{i, t}=1, Y_{k, t}=1 \mid D_{i, k, t}\right] / \mathbf{P}\left[Y_{i, t}=\right.\right.$ $\left.\left.0, Y_{k, t}=0 \mid D_{i, k, t}\right]\right)$ in two different ways; equating them yields

$$
\begin{aligned}
\log \frac{\mathbf{P}\left[Y_{i, t}=1 \mid Y_{k, t}=1, D_{i, k, t}\right]}{\mathbf{P}\left[Y_{i, t}=0 \mid Y_{k, t}=1, D_{i, k, t}\right]} & +\log \frac{\mathbf{P}\left[Y_{k, t}=1 \mid Y_{i, t}=0, D_{i, k, t}\right]}{\mathbf{P}\left[Y_{k, t}=0 \mid Y_{i, t}=0, D_{i, k, t}\right]} \\
& =\log \frac{\mathbf{P}\left[Y_{k, t}=1 \mid Y_{i, t}=1, D_{i, k, t}\right]}{\mathbf{P}\left[Y_{k, t}=0 \mid Y_{i, t}=1, D_{i, k, t}\right]}+\log \frac{\mathbf{P}\left[Y_{i, t}=1 \mid Y_{k, t}=0, D_{i, k, t}\right]}{\mathbf{P}\left[Y_{i, t}=0 \mid Y_{k, t}=0, D_{i, k, t}\right]}
\end{aligned}
$$

Use of the model equation to evaluate each of these four logarithms (use the equation for $(i, k)$ when predicting $Y_{i, t}$ and the equation for $(k, m)$ when predicting $\left.Y_{k, t}\right)$ yields an equation in which all terms cancel but one-leaving $\beta_{1}=0$.

Since [2] and [3] use these same kinds of models, those papers share these same problems. The paper [4] uses mostly linear regressions rather than logistic regressions. These regression equations are almost the same, but now the response variable, $Y_{i, t}$, is the number of days per week $i$ is lonely at time $t$. In [4], C\&F posit that each tie $(i, j)$ at time $t \in\{6,7\}$ satisfies

$$
Y_{i, t}=\alpha+\left(\beta_{1} Y_{j, t}+\beta_{2} Y_{j, t-1}\right)+\beta_{3} Y_{i, t-1}+\gamma_{7} W_{7}(t)+\delta_{1} A_{i, t}+\delta_{2} F_{i}+\delta_{3} E_{i, t}+\varepsilon_{i, j, t},
$$

where $\varepsilon_{i, j, t}$ is an error term that (presumably) is independent of the other variables on the right-hand side, as well as of all variables at time $t-1$, and has mean 0 .

This model also contradicts the data unless $\beta_{1}=\beta_{2}=0$. To see this, take the expectation conditional on time $t-1$ and thereby eliminate the error terms. If $(i, j),(j, i),(j, k),(k, j) \in T_{t}$, where $k \neq i$, then we get 4 equations for the 3 unknowns, these 3 unknowns having the form $\mathbf{E}\left[Y_{m, t} \mid\right.$ time $\left.t-1\right]$ for $m=i, j, k$. Since the 4 equations have a solution, we get an equation that the data at time $t-1$ must satisfy. Some algebra shows that to prevent a contradiction, this equation implies that $\beta_{1}=\beta_{2}=0$.

\section{Model Estimation}

C\&F estimate their 12 coefficients via a method known as generalized estimating equations (GEE). This method is designed for repeated measures or other sorts of dependencies, but itself comes with some assumptions [23, Theorem 1]. One assumption is independence among subjects or among groups of subjects.

\footnotetext{
${ }^{3}$ One might also make the probabilities on the left-hand side of the equation conditional on the other covariates, but we shall treat them as non-random for brevity.

${ }^{4}$ In a different context, this same conclusion, that $\beta_{1}=0$, is proved in [21]; see also [22].
} 
Since C\&F have not clearly delineated their use of GEE, we must guess how they are using it from their descriptions such as "We used generalized estimating equations to account for multiple observations of the same ego across examinations and across ego-alter pairs. We assumed an independent working correlation structure for the clusters" [1] and "Models were estimated using a general estimating equation with clustering on the focal participant and an independent working covariance structure" [4]. This seems to mean that all the measurements on each FP were a single cluster, or group. If so, however, then these groups are not independent; indeed, C\&F wish to make conclusions about their dependencies. The "working covariance" seems to relate to the different measurements in time. Yet GEE relies on having a large number of independent groups.

Moreover, there is a peculiar twist to the equations of C\&F's models due to the interest in the social network: the dependent variables (the $Y_{i, t}$ ) appear not only on the left-hand sides of the equations, but also on the right-hand sides. This is not part of the literature regarding GEE, at least to our knowledge. It is possible that C\&F's estimation method could work when the model holds, but we would need to see mathematical proof or relevant simulation results.

In any case, we do have enough information to know that GEE does not work for C\&F's model: Since the model implies that $\beta_{1}=0$, yet $\mathrm{C} \& \mathrm{~F}$ estimate that $\beta_{1} \neq 0$, it follows that their estimation method is faulty.

\section{The Role of Review}

Both of C\&F's first two papers were published in the world's top medical journal, the New Engl. J. Med. Their third paper was published in BMJ, another very highly respected medical journal. Their fourth paper was published in the J. Pers. Soc. Psychol., a top journal and the flagship journal of the American Psychological Association. After we had completed our analysis of those four papers, two more based on the same data appeared: the fifth [24] in Ann. Intern. Med., again a very highly respected journal, and the sixth [25] in Mol. Psychiatry, a top journal in psychiatry. We leave as an exercise to the reader to spot in these last two papers the same errors we have recounted here.

Given the fundamental errors we have described, what can we conclude about the process of peer review at these top journals? Altman [26], currently the senior statistics editor at BMJ, gave a personal account as a statistical reviewer of submissions to medical journals, as well as a table summarizing some studies on the quality of statistics in published medical articles. His bleak assessment: "The main reason for the plethora of statistical errors is that the majority of statistical analyses are performed by people with an inadequate understanding of statistical methods. They are then peer reviewed by people who are generally no more knowledgeable. Sadly, much research may benefit researchers rather more than patients, especially when is carried out primarily as a ridiculous career necessity."

Problems with peer review have long been known and several remedies have been proposed. One remedy has even been shown to fail: see [27]. We propose a new solution below, based partly on our experiences in getting the present critique published. One can find several anecdotal reports on the web about the policies of top scientific journals regarding critiques, but we are not aware of any study of the issue. Our experiences matched the anecdotes we saw and seem informative.

We first submitted our critique to the New Engl. J. Med., but it was rejected without peer review. The journal declined to give a reason when asked. We next submitted to BMJ, but it was again rejected without peer review. This journal did, however, volunteer that "We decided your paper was probably better placed in a more specialist journal." It is interesting to note that the same issue of BMJ that published [3] also published the critique [15]. The cover of that issue, in fact, was devoted to those two articles. In contrast to BMJ's decision, the general-interest online newsmagazine Slate published an article [28] on our critique the same month we submitted our paper. An delightful coda is that a few months later, BMJ published an editorial called "Inadequate post-publication review of medical research" [29].

After these rejections by the New Engl. J. Med. and BMJ, we approached three top journals who did not publish any of C\&F's studies, JAMA, Lancet, and Proc. Natl. Acad. Sci.. All were uninterested in our critique since they do not publish critiques of articles they did not originally publish. The section of $J$. 
Pers. Soc. Psychol. that published [4] does not publish critiques even of papers they have published, unless accompanied by new data.

Following on this educational venture, we submitted to a statistics journal that specializes in reviews, Stat. Sci. Five months later they had 3 referee reports. The first two recommended publication after revisions (e.g., "an important critique" and "well worth publishing"), while the third, though agreeing with our critiques, said that C\&F's work was insufficiently important to warrant publication of a critique in Stat. Sci. Two months after getting these reports, the editor made his decision: rejection, allowing for resubmission if we made the tone more neutral and changed the focus, perhaps to "editorial decision making standards in medical journals", as suggested by the third referee.

Methodological journals abound, but their cautions and recommendations are largely ignored [30]. Indeed, "in a process well documented by Blalock and Duncan, positivist sociology, like so many other professions, has tended to become immune to the recognition of flaws in its work" [31]. Given the above considerations, it may help to have a journal specifically devoted to critiques. ${ }^{5}$ This would not only allow others to know more about which studies are trustworthy, but could also have the salutary effect of encouraging researchers to pay extra attention to their methods lest they be publicly critiqued.

\section{Conclusions}

We begin by summarizing the major problems with C\&F's studies:

(1) The data are not available to others.

(2) The unavailable data are sparse for friendships.

(3) The models used to analyze the sparse data contradict the data and the conclusions.

(4) The method used to estimate the dubious models does not apply.

(5) The statistical significance tests from the questionable estimates do not show the proposed differences.

(6) The wrongly proposed differences do not distinguish among homophily, environment, and induction.

(7) Associations at a distance are better explained by homophily than by induction.

How did these errors arise and pass inspection? We believe that one major reason is that, as many before us have said, statistical assumptions are routinely made when they are unlikely to hold. The motivation for making assumptions is the hope of overcoming the limitations of observational data, especially for causal inference. In any particular case, some of those limitations are known, while others are unknown. Yet viewing observational data through the lens of statistical modeling produces new biases, generally unknown and mostly unacknowledged, lurking in mathematical thickets. Unfortunately, controlling for selection effects and other confounders is extraordinarily difficult in observational studies [32]; this is the main reason that observational studies are regarded with skepticism. Indeed, as demonstrated with the well-known studies concerning hormone-replacement therapy, it was impossible to control the observational studies to get the same effects as the experiments [33,34]. Observational studies often lead to publications whose causal conclusions contradict one another or are contradicted by experiments [32, 35, 36]; this is a natural consequence of poor methodology.

Some investigators have found much better data, even if not perfect, to assess causal effects in social networks. For example, [37, 38] look at random assignments of college freshmen. This is promising, although both these cited studies make assumptions about what their data look like without presenting that data, nor telling us why the data fit their models. It is not uncommon to subject experimental data to statistical modeling, but this, too, likely leads to biases [39]. Of course, small-scale experiments could be initiated to see what the effects of intervention actually are. Since the collection of good data is usually very hard and expensive, most papers substitute for it by statistical modeling [30]. "But progress is unlikely," wrote Summers [40], "as long as [we] require the armor of a stochastic pseudo-world before doing battle with evidence from the real one."

We can learn $[19,41]$ from others' experiences of modeling observational data, but there is also an $a$ priori reason to distrust modeling in the absence of the ability to confirm or deny the results: Rarely can

${ }^{5}$ We thank Elchanan Mossel for this idea. 
one know whether the needed assumptions are correct - otherwise, they wouldn't be assumptions [42]. Yet they are crucial to analysis when modeling. In order to bring these issues to light in published research, analysts who model should, at a minimum, state their models fully and explicitly, complete with equations and assumptions. Similarly, analysts should clearly report their estimation methods and the assumptions behind them. Such clarity will not only aid readers and reviewers, it may also alert authors to mistakes in reasoning before they are committed to print. It is also wise to bear in mind that technical fixes (such as adding a lagged obesity term to a logistic regression model to account for homophily) work only for technical problems, not for fundamental issues [19]. Other recommendations for better statistical practice can be found in [41, 43] (among hundreds of other articles).

Medical journals often publish articles and editorials measuring and bemoaning the quality of evidence in medicine [44, 45, 43]. The situation is so bad that a recent study [46] of the medical literature was titled "Why Most Published Research Findings Are False" ${ }^{6}$; the author later was appointed to the Stanford University School of Medicine. These concerns have spread to the popular media: see [47, 48, 49]. Of course, the nature of almost all medical evidence is statistical.

Medicine has many comrades who share its concern over the misuse of statistics. Since Keynes [50, 51], there have been some social scientists who have decried tendencies in their fields towards statistical idolatry; Keynes used the phrases "black magic" and "statistical alchemy". See [19, Chap. 10] for a review of some of the vast critical literature and, e.g., [52,31] and the references there. Among the concerned was Otis Dudley Duncan, one of the most important quantitative sociologists of the last century. More than 25 years ago, he wrote [53, p. 226]:

Coupled with downright incompetence in statistics, paradoxically, we often find the syndrome that I have come to call statisticism: the notion that computing is synonymous with doing research, the naive faith that statistics is a complete or sufficient basis for scientific methodology, the superstition that statistical formulas exist for evaluating such things as the relative merits of different substantive theories or the "importance" of the causes of a "dependent variable"; and the delusion that decomposing the covariations of some arbitrary and haphazardly assembled collection of variables can somehow justify not only a "causal model" but also, praise the mark, a "measurement model." There would be no point in deploring such caricatures of the scientific enterprise if there were a clearly identifiable sector of social science research wherein such fallacies were clearly recognized and emphatically out of bounds.

Duncan hoped that criticism of such "abuses ... might lead to something like the famed Flexner report of 1910 that put the spotlight on the miserable state of medical education at that time." [53, p. 227] Indeed, an obvious "cure" for poor statistical practice is to improve statistics education. While there is widespread agreement on the need for statistical literacy among the populace at large, efforts to improve the statistical competence of those who become practitioners receive less attention.

We see the problems with existing statistics education as follows. Although most statistics courses mention the importance of the assumptions behind the techniques they present, few devote much time to this topic. Such lack of attention is especially prevalent in more advanced courses taught in a variety of disciplines $^{7}$, yet the assumptions behind more advanced techniques are considerably more subtle than those in elementary courses. Most students, who are generally practically minded, learn not to question whether the assumptions hold in practical situations - or, at least, students do not learn to question the assumptions. Many such students later become practitioners and, often, educators themselves: more statistics is taught outside statistics departments than within. In the face of academic pressure to publish papers, assumptions become inconvenient and further marginalized, even though all assent to their importance. Thus, Blalock [30] wrote of his profession, sociology:

$[\mathrm{O}]$ ne finds a large number of journal articles that briefly discuss the measurement of selected variables, that also admit to the possibility of errors, but that then effectively announce to the reader that the

\footnotetext{
${ }^{6}$ Although the title may be correct, the article did not provide sufficient empirical evidence to establish it.

7 Those who feel that most textbooks do pay serious attention to assumptions are urged to compare those books to [54, 19] and, especially, to compare their exercises. Especially useful are exercises that present possible mistakes in the published literature, while asking students whether the statistical conclusions were justified.
} 
subsequent empirical analysis and related interpretations will proceed as though there were absolutely no measurement errors whatsoever!

This is but one illustration of the more general point that methodological ideas are adopted when it is relatively easy and costless to do so, but that they are resisted or totally ignored when it is to the investigator's vested interest to do so.

It is to counteract these natural tendencies that we urge much greater attention to questioning assumptions.

Flawed statistical models are not limited to medicine and academia. For example, the current economic afflictions are partly due to flawed models: see [55, Section II.D], [56, pp. 16, 28, 44, 149] and [57, pp. 288ff]. An examination of statistics education, as Duncan suggested, is overdue. Educators need not wait for any report, however, before we ourselves teach critical thinking [54, 19].

Acknowledgements. I am grateful to Abie Flaxman, Jason Fletcher, Elizabeth Housworth, Janet Macher, Roger Purves, Philip Stark, and Duncan Watts for helpful conversations, suggestions, and remarks. I also thank the referees for useful suggestions and references.

\section{Appendix A. Directionality Table}

The overlapping confidence intervals for directional coefficient estimates were shown in Figure 1 . The actual numbers are given here in Table 1 . They are reported both as probability estimates with CIs and as coefficient estimates with SEs, for the following reason. Logistic regression models transform numbers on the right-hand side into probabilities on the left-hand side. However, one must choose values for every covariate in order to get a probability. Even when one varies a right-hand side coefficient in order to see how the uncertainty in its estimate transforms into an uncertainty in probability, one must choose values for all the covariates because of the non-linear nature of the transformation. Since this transformation depends on the values chosen for the covariates, there is in principle one probability and one CI for each FP. What C\&F report instead are probabilities and CIs when the covariates are assigned their mean values over the population. This doesn't represent anyone (e.g., the gender variable is $1 / 2$, while for a person, it is either 0 or 1). Thus, such probabilities and CIs are only a vague kind of average of the individual probabilities and CIs. This is a well-known difficulty with logistic regression models.

\begin{tabular}{|c|ccc|}
\hline Source & FP $\leftrightarrow$ LP & FP $\rightarrow$ LP & LP $\rightarrow$ FP \\
\hline [1], p. 376 & $171 \%[59 \%, 326 \%]$ & $57 \%[6 \%, 123 \%]$ & $13 \%[-28 \%, 68 \%]$ \\
[1], suppl. p. 3 & $1.19(0.33)$ & $0.52(0.23)$ & $0.11(0.28)$ \\
[16], p. 1401 & & $0.033(0.014)$ & $0.002(0.014)$ \\
[2], pp. 2254, 2256 & $43 \%[1 \%, 69 \%]$ & $36 \%[12 \%, 55 \%]$ & $15 \%[-35 \%, 50 \%]$ \\
[2], suppl. p.18 & $0.66(0.33)$ & $0.51(0.19)$ & $0.21(0.27)$ \\
[3], p. 6 & $63 \%[12 \%, 148 \%]$ & $25 \%[1 \%, 57 \%]$ & $12 \%[-13 \%, 47 \%]$ \\
[3], suppl. p. 9 & $2.07(0.79)$ & $0.70(0.34)$ & $0.32(0.41)$ \\
[4], pp. 983-984 & $0.41(0.13)$ & $0.29(0.11)$ & $0.35(0.30)$ \\
\hline
\end{tabular}

Table 1. Directional differences for friendship ties. Key: FP $\leftrightarrow$ LP means mutual friendship; FP $\rightarrow$ LP means FP named LP; LP $\rightarrow$ FP means LP named FP; FP = ego; LP = alter. [Reported 95\% CIs] and (reported SEs). 


\section{Appendix B. Further Lack of Statistical Significance}

Section 2 showed that C\&F's directional analysis was flawed by lack of statistical significance (among other flaws). This same flaw occurs in other comparisons C\&F make. For example, [3] states that "Coresident spouses who become happy increase the probability their spouse is happy by $8 \%$ ( $0.2 \%$ to $16 \%)$, while noncoresident spouses have no significant effect." That is, C\&F say that coresident spouses have an effect, while non-coresident spouses do not. The mistake is that this is based on the second covariate (non-coresident spouses) having a coefficient that is statistically non-significant: the coefficient translates to a probability of $2 \%$ with a CI so large, $[-18 \%, 31 \%]$, that it engulfs the CI for the first covariate. Thus, the difference between the two coefficients cannot be said to be statistically significant. Again, C\&F's methods do not permit a comparison between the importance of these two covariates. Similar examples are listed in Table 2 .

\begin{tabular}{|c|cc|}
\hline Source & Covariate 1 & Covariate 2 \\
\hline$[1]$, p. 376 & same sex $71 \%[13 \%, 145 \%]$ & opposite sex $-9 \%[-62 \%, 117 \%]$ \\
{$[1]$, p. 376} & M same sex $100 \%[26 \%, 197 \%]$ & F same sex $38 \%[-39 \%, 161 \%]$ \\
{$[2]$, p. 2254} & FP college $57 \%[29 \%, 75 \%]$ & LP no college $4 \%[-67 \%, 43 \%]$ \\
{$[2]$, p. 2254} & LP college $55 \%[26 \%, 74 \%]$ & LP no college $4 \%[-67 \%, 43 \%]$ \\
{$[2]$, p. 2254} & both college $61 \%[28 \%, 81 \%]$ & LP no college $4 \%[-67 \%, 43 \%]$ \\
{$[2]$, pp. 2255-2256, suppl. p. 31} & moderate smoking, various & heavy smoking, various \\
{$[2]$, suppl. p. 15} & late period $-70.89(35.9)$ & early period $11.49(13.3)$ \\
{$[3]$, p. 6} & nearby friend $25 \%[1 \%, 57 \%]$ & distant friend $-3 \%[-15 \%, 10 \%]$ \\
{$[3]$, pp. $6-7$} & coresident spouse $8 \%[0.2 \%, 16 \%]$ & non-coresident spouse $2 \%[-18 \%, 31 \%]$ \\
{$[3]$, pp. $6-7$} & nearby sibling $14 \%[1 \%, 28 \%]$ & distant sibling $2 \%[-3 \%, 8 \%]$ \\
\hline
\end{tabular}

Table 2. Statistically insignificant comparisons. Covariate 1 is statistically significant, while Covariate 2 is not. [Reported 95\% CIs] and (reported SEs).

Even when not making comparisons, $\mathrm{C} \& \mathrm{~F}$ sometimes conclude that a number is 0 when their methods tell them only that they cannot distinguish it statistically from 0. For example, [1] states that "Obesity in a sibling of the opposite sex did not affect the chance that the other sibling would become obese." Other such examples are listed in Table 3.

\begin{tabular}{|c|c|}
\hline Source & Covariate \\
\hline [1], p. 376 & opposite sex sibling $27 \%[3 \%, 54 \%]$ \\
[2], suppl. p. 15 & early current centrality $2.20(91.31)$ \\
[2], suppl. p. 15 & late current centrality -138.00 (156.00) \\
[3], p. 6, suppl. p. 7 & additional unhappy alter $-0.06(0.03)$ \\
[3], p. 7, suppl. p. 10 & coworkers -0.29 (0.16) \\
\hline
\end{tabular}

Table 3. Statistically insignificant conclusions. Covariate coefficient is reported not statistically significant, but the authors treat it as 0, even though the CI was not close to 0. [Reported 95\% CI] and (reported SEs). 


\section{REFERENCES}

[1] N. A. Christakis and J. H. Fowler, "The spread of obesity in a large social network over 32 years," N. Engl. J. Med., vol. 357, pp. 370-379, 2007.

[2] N. A. Christakis and J. H. Fowler, "The collective dynamics of smoking in a large social network," N. Engl. J. Med., vol. 358, pp. 2249-2258, 2008.

[3] J. H. Fowler and N. A. Christakis, "Dynamic spread of happiness in a large social network: longitudinal analysis over 20 years in the Framingham Heart Study," BMJ, vol. 337, p. a2338, 2008. doi:10.1136/bmj.a2338.

[4] J. T. Cacioppo, J. H. Fowler, and N. A. Christakis, "Alone in the crowd: the structure and spread of loneliness in a large social network," J. Pers. Soc. Psychol., vol. 97, no. 6, pp. 977-991, 2009.

[5] G. Kolata, "Find yourself packing it on? Blame friends," New York Times, p. 1, July 26, 2007.

[6] P. Belluck, "Strangers may cheer you up, study says," New York Times, December 4, 2008.

[7] N. A. Christakis and J. H. Fowler, Connected: The Surprising Power of Our Social Networks and How They Shape Our Lives. New York: Little, Brown and Co., 2009.

[8] C. Thompson, "Are your friends making you fat?," New York Times, September 10, 2009.

[9] A. Steptoe and A. V. D. Roux, "Editorial: Happiness, health, and social networks," BMJ, vol. 337, p. a2781, 2008. doi:10.1136/bmj.a2781.

[10] M. J. Morgan, "The contagion of happiness," BMJ, 2009. http://www.bmj.com/cgi/eletters/337/dec04_2/a2338\#207624.

[11] C. R. Shalizi and A. C. Thomas, "Homophily and contagion are generically confounded in observational social network studies," Sociol. Method. Res., 2011. To appear.

[12] G. King, "Replication, replication," PS: Polit. Sci. Polit., vol. 28, no. 3, pp. 444-452, 1995.

[13] K. Baggerly and K. Coombes, "Deriving chemosensitivity from cell lines: Forensic bioinformatics and reproducible research in high-throughput biology," Ann. Appl. Stat., vol. 3, no. 4, pp. 1309-1334, 2009.

[14] E. Cohen-Cole and J. M. Fletcher, "Is obesity contagious? Social network vs. environmental factors in the obesity epidemic," J. Health Econ., vol. 27, no. 5, pp. 1382-1387, 2008.

[15] E. Cohen-Cole and J. M. Fletcher, "Detecting implausible social network effects in acne, height, and headaches: longitudinal analysis," BMJ, vol. 337, p. a2533, 2008. doi:10.1136/bmj.a2533.

[16] J. H. Fowler and N. A. Christakis, "Estimating peer effects on health in social networks," J. Health Econ., vol. 27, no. 5, pp. 1400-1405, 2008.

[17] H. Noel and B. Nyhan, "The 'unfriending' problem: The consequences of homophily in friendship retention for causal estimates of social influence," Soc. Networks, 2011. To appear.

[18] P. Sainsbury, "Commentary: Understanding social network analysis," BMJ, vol. 337, p. a1957, 2008. doi:10.1136/bmj.a1957.

[19] D. A. Freedman, Statistical Models: Theory and Practice. Cambridge: Cambridge University Press, revised ed., 2009. With a foreword by David Collier, Jasjeet Singh Sekhon and Philip B. Stark.

[20] N. A. Christakis and J. H. Fowler, "Examining dynamic social networks and human behavior," 2010. Preprint.

[21] J. J. Heckman, "Dummy endogenous variables in a simultaneous equation system," Econometrica, vol. 46, no. 4, pp. 931959, 1978.

[22] E. Tamer, "Incomplete simultaneous discrete response model with multiple equilibria," Rev. Econ. Studies, vol. 70, pp. 147$165,2003$.

[23] K. Y. Liang and S. L. Zeger, "Longitudinal data analysis using generalized linear models," Biometrika, vol. 73, no. 1, pp. 13-22, 1986.

[24] J. N. Rosenquist, J. Murabito, J. H. Fowler, and N. A. Christakis, "The spread of alcohol consumption behavior in a large social network," Ann. Intern. Med., vol. 152, no. 7, pp. 426-433, 2010.

[25] J. N. Rosenquist, J. H. Fowler, and N. A. Christakis, "Social network determinants of depression," Mol. Psychiatry, vol. 16, no. 3, pp. 273-281, 2011.

[26] D. G. Altman, "Statistical reviewing for medical journals," Stat. Med., vol. 17, no. 23, pp. 2661-2674, 1998.

[27] F. Fidler, N. Thomason, G. Cumming, S. Finch, and J. Leeman, "Editors can lead researchers to confidence intervals, but can't make them think," Psychol. Sci., vol. 15, no. 2, pp. 119-126, 2004.

[28] D. Johns, "Everything is contagious: Doubts about the social plague stir in the human superorganism," Slate, April 8, 2010. http://www.slate.com/id/2250102/.

[29] D. L. Schriger and D. G. Altman, "Inadequate post-publication review of medical research," BMJ, vol. 341, p. c3803, 2010. doi:10.1136/bmj.c3803.

[30] H. M. Blalock, Jr., "The real and unrealized contributions of quantitative sociology," Am. Sociol. Rev., vol. 54, no. 3, pp. 447-460, 1989.

[31] B. Baldus, "Positivism's twilight?," Can. J. Sociol., vol. 15, no. 2, pp. 149-163, 1990.

[32] J. P. A. Ioannidis, "Contradicted and initially stronger effects in highly cited clinical research," JAMA, vol. 294, no. 2, pp. 218-228, 2005.

[33] D. B. Petitti and D. A. Freedman, "Invited commentary: How far can epidemiologists get with statistical adjustment?," Am. J. Epidemiol., vol. 162, pp. 415-418, 2005.

[34] D. A. Freedman and D. B. Petitti, "Hormone replacement therapy does not save lives: Comments on the Women's Health Initiative," Biometrics, vol. 61, pp. 918-920, 2005. 
[35] W. Maziak, "The triumph of the null hypothesis: epidemiology in an age of change," Int. J. Epidemiol., vol. 38, no. 2, pp. 393-402, 2009.

[36] G. Taubes, "Epidemiology faces its limits," Science, vol. 269, pp. 164-169, 1995.

[37] B. Sacerdote, "Peer effects with random assignment: Results for Dartmouth roommates," Q. J. Econ., vol. 116, no. 2, pp. 681-704, 2001.

[38] S. E. Carrella, M. Hoekstrab, and J. E. West, "Is poor fitness contagious?: Evidence from randomly assigned friends," J. Public Econ., vol. 95, no. 7-8, pp. 657-663, 2011.

[39] D. A. Freedman, "On regression adjustments in experiments with several treatments," Ann. Appl. Stat., vol. 2, pp. 176-196, 2008.

[40] L. H. Summers, "The scientific illusion in empirical macroeconomics," Scand. J. Econ., vol. 93, no. 2, pp. 129-148, 1991. Proceedings of a Conference on New Approaches to Empirical Macroeconomics.

[41] D. A. Freedman, "Survival analysis: A primer," Am. Stat., vol. 62, pp. 110-119, 2008.

[42] D. A. Freedman, "Oasis or mirage?," CHANCE Mag., vol. 21, no. 1, pp. 59-61, 2008.

[43] D. G. Altman, "Poor-quality medical research: What can journals do?," JAMA, vol. 287, no. 21, pp. 2765-2767, 2002. doi:10.1001/jama.287.21.2765.

[44] S. A. Glantz, "Biostatistics: how to detect, correct and prevent errors in the medical literature," Circulation, vol. 61, no. 1, pp. $1-7,1980$.

[45] R. Smith, "Where is the wisdom? The poverty of medical evidence," Brit. Med. J., vol. 303, no. 6808, pp. 798-799, 1991.

[46] J. P. A. Ioannidis, "Why most published research findings are false," PLoS Med., vol. 2, no. 8, p. e124, 2005. doi:10.1371/journal.pmed.0020124.

[47] T. Siegfried, "Odds are, it's wrong: Science fails to face the shortcomings of statistics," Science News, vol. 177, no. 7, pp. 26-29, March 27, 2010.

[48] D. H. Freedman, "Lies, damned lies, and medical science," The Atlantic, Nov. 2010.

[49] S. Begley, "Why almost everything you hear about medicine is wrong," Newsweek, January 24, 2011.

[50] J. M. Keynes, "Professor Tinbergen's method," Econ. J., vol. 49, pp. 558-568, 1939.

[51] J. M. Keynes, "Comment [on Tinbergen's reply]," Econ. J., vol. 50, pp. 154-156, 1940.

[52] G. Gigerenzer, "Mindless statistics," J. Socio-Econ., vol. 33, no. 5, pp. 587-606, 2004.

[53] O. D. Duncan, Notes on Social Measurement: Historical and Critical. New York: Russell Sage Foundation, 1984.

[54] D. A. Freedman, R. Pisani, and R. Purves, Statistics. New York: W. W. Norton \& Co., 4th ed., 2007.

[55] J. E. Stiglitz, "Lessons from the global financial crisis of 2008," Seoul J. Econ., vol. 23, no. 3, pp. 321-339, 2010.

[56] Financial Crisis Inquiry Commission, The Financial Crisis Inquiry Report: Final Report of the National Commission on the Causes of the Financial and Economic Crisis in the United States. PublicAffairs, 2011.

[57] Permanent Subcommittee on Investigations, Wall Street and the Financial Crisis: Anatomy of a Financial Collapse. United States Senate, 2011. Majority and minority staff report. 